\title{
FERRAMENTA DE SIMULAÇÃO COMPUTACIONAL PARA EVOLUÇÃO DAS TEMPERATURAS INTERNAS DO REFRATÁRIO DE UM FORNO DE TRATAMENTO TÉRMICO DE TUBOS DE AÇO*
}

\author{
Arnaud-Guilhem de Loze \\ Luis Fernando Oliveira Rodrigues ${ }^{2}$ \\ Lis Nunes Soares ${ }^{3}$ \\ Stephano Joviano Miranda ${ }^{4}$
}

\section{Resumo}

Este artigo apresenta uma ferramenta de simulação computacional desenvolvida com o objetivo de auxiliar a adoção de melhores práticas de controle do forno durante períodos sem produção. O sistema desenvolvido permite simular as temperaturas do revestimento refratário de um forno de tratamento térmico de tubos em função da evolução da temperatura da atmosfera interna em diferentes cenários. O simulador foi desenvolvido baseado em um modelo matemático dinâmico validado, que utiliza um sistema de equações de transferência de calor discretizadas. Realizou-se a validação desta ferramenta computacional com a utilização de dados reais de uma campanha de medição de temperaturas. A comparação dos resultados apresentou pequeno erro entre simulação e valores reais coletados. A utilização desta ferramenta auxiliou a definição de estratégias de controle do forno durante períodos improdutivos, possibilitando redução do consumo de energia e retomada da produção com garantia da qualidade do produto.

Palavras-chave: Transferência de calor; Fornos de tratamento térmico; Revestimento refratário.

\section{COMPUTER-BASED SIMULATION TOOL OF STEEL TUBES HEAT TREATMENT FURNACE REFRACTORY MATERIALS TEMPERATURE EVOLUTIONS}

\begin{abstract}
This paper presents a computer-based tool developed in order to assist best practices of furnace control, during periods without production. The system simulates refractory materials temperature evolutions of a steel pipes heat treatment furnace in function of internal atmosphere temperature. The tool is based on a validated mathematic dynamic model, which uses a discretized method for heat transfer equations system. Validation was performed using data from a measurement campaign. Comparison between the model results and measurements shows small relative differences. The tool enables to define furnace temperature conditions, during no production period, to save energy and return production process with product quality.

Keywords: Heat transfer; Heat treatment furnace; Refractory materials.

1 Engenheiro de Energia, Ecole Centrale Paris, Paris, França.

2 Estudante de engenharia mecânica, estagiário, Área de Tecnologia Térmica/Superintendência Geral de Produção, Vallourec Tubos do Brasil S.A., Belo Horizonte, Minas Gerais, Brasil.

3 Engenheira Mecânica, Doutora, Engenheira de Processos Sênior, Área de Tecnologia Térmica/Superintendência Geral de Produção, Vallourec Tubos do Brasil S.A., Belo Horizonte, Minas Gerais, Brasil.

4 Estudante de engenharia mecânica, estagiário, Área de Tecnologia Térmica/Superintendência Geral de Produção, Vallourec Tubos do Brasil S.A., Belo Horizonte, Minas Gerais, Brasil.
\end{abstract}




\section{INTRODUÇÃO}

Fornos são equipamentos essenciais para o processo de laminação e tratamento térmico de tubos de aço sem costura. Visando garantia da qualidade do produto e redução de consumo de energia, é importante o entendimento detalhado dos processos de transferência de calor que ocorrem nestes equipamentos. Este artigo apresenta uma ferramenta computacional desenvolvida para a simulação da evolução de temperaturas em camadas internas do revestimento refratário, durante diferentes cenários, em função da evolução de temperaturas da atmosfera interna do forno ao longo do tempo.

Este simulador foi desenvolvido com o objetivo de auxiliar a adoção de melhores práticas de controle do forno durante períodos sem produção, visando redução do consumo energético com garantia da qualidade dos tubos a serem enfornados no início do processo produtivo. O desenvolvimento deste sistema foi baseado em um modelo matemático validado desenvolvido por de Loze ${ }^{[1]}$. Basicamente, este modelo utiliza equações de transferência de calor discretizadas para o cálculo da evolução de temperaturas em determinados pontos internos do material refratário. A aplicação do modelo matemático em um sistema de cálculo computacional proporcionou um caráter prático ao estudo desenvolvido, facilitando assim, sua utilização para simulação e análise da condição de temperaturas do forno em períodos fora do regime de trabalho permanente. A aplicação prática do modelo matemático na ferramenta de simulação alcançou os objetivos almejados.

\section{MATERIAIS E MÉTODOS}

Para o desenvolvimento de uma ferramenta computacional prática para a simulação da evolução de temperaturas em determinados pontos internos do revestimento refratário do forno em questão, exigiu-se a aplicação do modelo matemático de transferência de calor em linguagem de programação VBA, e a validação do mesmo a partir de uma análise comparativa entre os resultados do programa e dados reais coletados.

\subsection{Forno de tratamento térmico}

Trata-se de um forno retangular de vigas caminhantes, com revestimento refratário interno composto por cimento refratário na região da soleira e nas paredes, até metade inferior de sua altura; e com revestimento refratário de fibra cerâmica na região do teto e nas paredes, na metade superior de sua altura. Externamente, o forno é revestido por chapas de metal. O combustível utilizado é o gás natural.

\subsection{Modelo matemático validado}

O modelo matemático, de Loze ${ }^{[1]}$, foi utilizado para a rotina de cálculo implementada no sistema de simulação computacional e as principais relações de transferência de calor, como apresentado na Figura 1, foram consideradas. Em relação ao forno e o ambiente externo, considerou-se o fluxo de calor por radiação e convecção entre teto e soleira e a atmosfera externa. Para o ambiente interno, foram consideradas a transferência de calor por radiação e convecção entre a atmosfera interna do forno (massa de gás e chama de combustão) e as superfícies internas da soleira e do teto; 
e também a condução de calor ao longo das paredes dos refratários de teto e soleira.

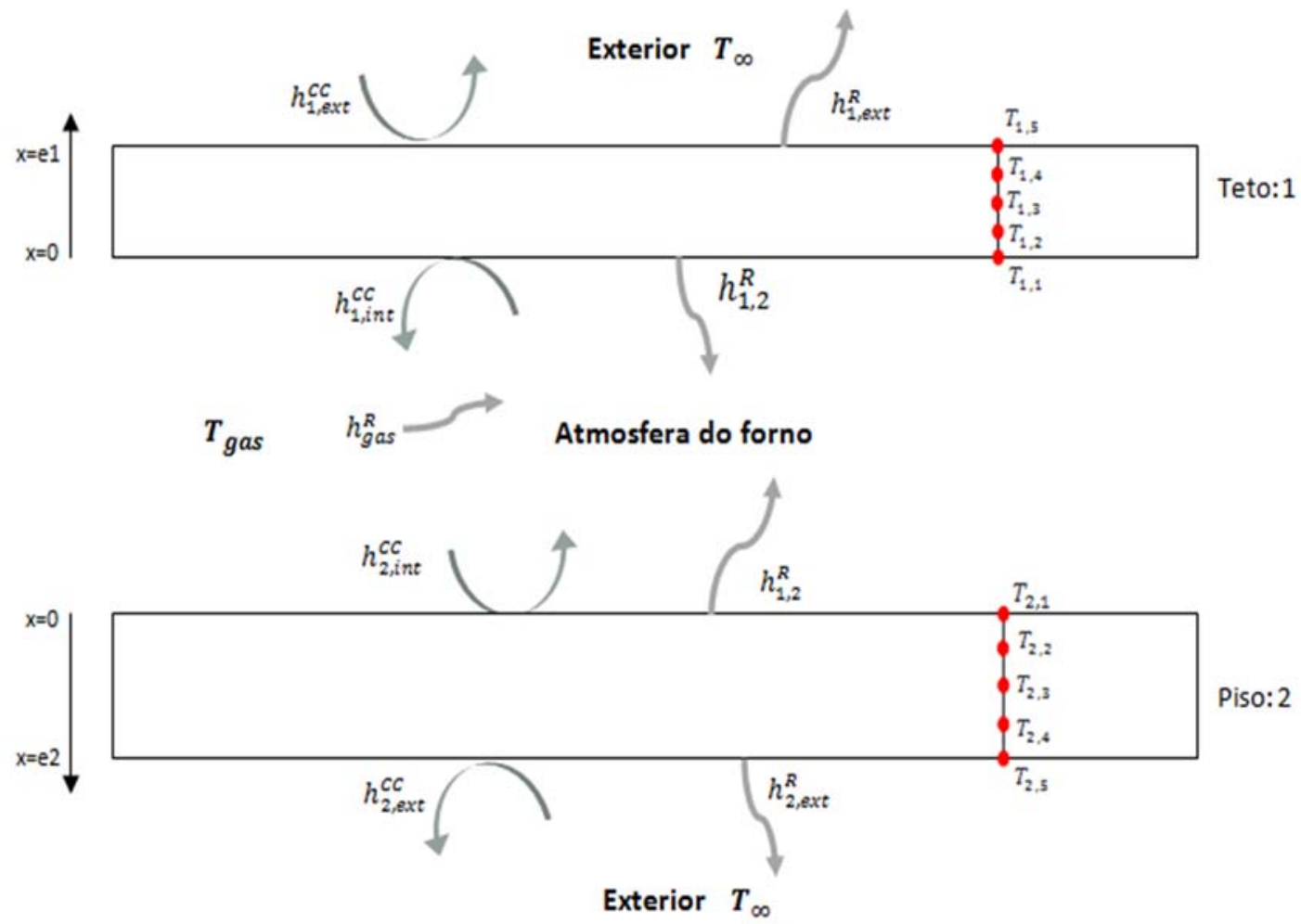

Figura 1. Relações de transferência de calor consideradas no modelo matemático.

A validação do modelo foi realizada através de uma campanha de medição de temperaturas. As medidas de temperatura durante a campanha foram comparadas com os valores de temperatura calculados sob a mesma condição.

\subsection{Estrutura de cálculo do simulador}

Para a resolução dos cálculos, adotou-se a discretização das equações em intervalos de tempo e de espaço (espessura do material refratário). A evolução da temperatura da atmosfera interna do forno ao longo do tempo, $T_{\text {gás, }}$ foi definida como dado de entrada e a partir desse parâmetro é calculada a evolução das temperaturas em camadas internas do revestimento refratário e o consumo de gás natural.

A estrutura de cálculo foi desenvolvida para abranger diferentes situações de evolução de temperatura, e para cada uma destas foram inseridos na rotina de cálculo parâmetros específicos, por exemplo, a relação entre consumo de gás natural $\left(\mathrm{Nm}^{3}\right)$ e taxa de aquecimento do forno $\left({ }^{\circ} \mathrm{C} / \mathrm{h}\right)$, observada a partir de análise profunda de dados reais retirados do sistema de controle. Os diferentes cenários considerados são:

- Elevação da temperatura do forno com mudança no regime de trabalho; denominado Ramp Up.

- Redução da temperatura do forno (redução controlada da temperatura de trabalho do forno) com alteração do regime de trabalho; denominado Ramp Down. 
- Ligamento do forno (após período desligado, com temperatura inicial próxima à temperatura ambiente), denominado Turn On.

- Desligamento do forno (corte do fornecimento de energia, resfriamento do equipamento de maneira não controlada), denominado Turn Off.

- Conservação do forno em temperatura constante, denominada Mantain.

Uma vez que cada cenário de evolução das temperaturas da atmosfera do forno apresenta características específicas, as opções oferecidas na estrutura de cálculo possibilitaram maior proximidade entre simulação e realidade.

\subsection{Campanha de medição de temperatura}

Para validação da ferramenta de simulação computacional foi realizada uma campanha de medições de temperaturas. Foram instalados termopares em camadas interiores de diferentes profundidades dos revestimentos refratários do teto e soleira do forno. Valores de temperatura foram coletados para um período de duas semanas, proporcionando uma boa visão geral dos diferentes cenários de evolução de temperatura. A Figura 2 apresenta o arranjo de instalação dos termopares. Os termopares são indicados na figura pela letra T e respectivo número de 1 a 3 . Sendo T1 o termopar de menor profundidade medida em relação à superfície externa do forno (face fria), e T3 o termopar de maior profundidade em relação à superfície externa (mais próximo à face quente do forno).
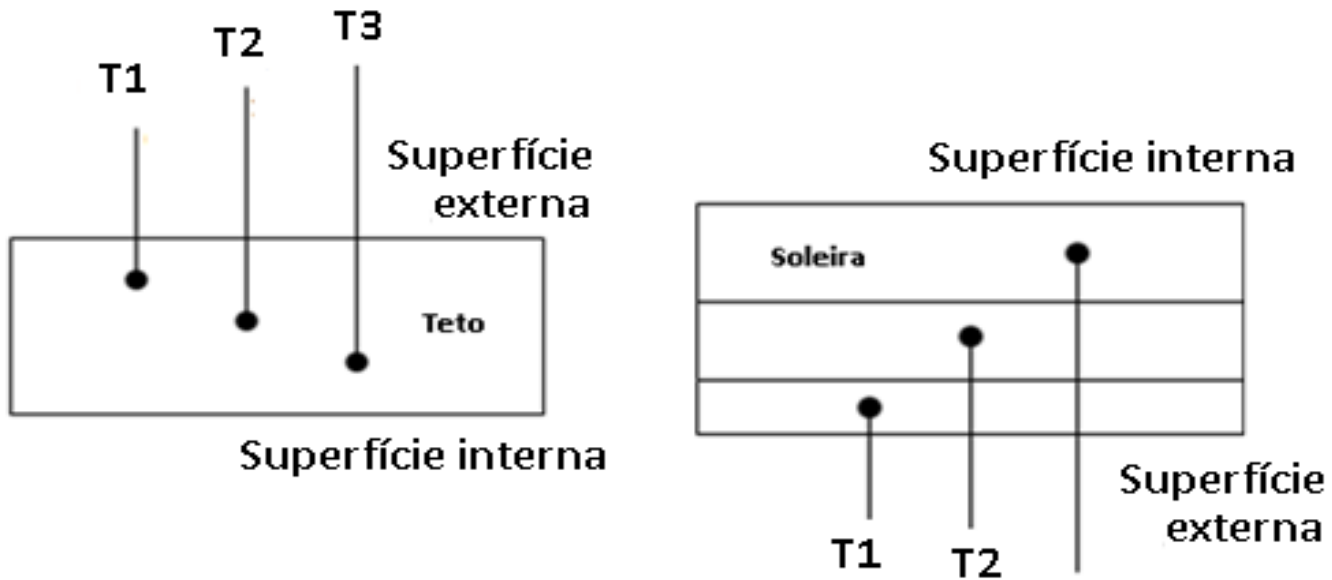

T3

Figura 2. Desenho esquemático dos pontos de instalação dos termopares no teto e soleira.

Os pontos para medição de temperatura foram definidos, levando em consideração características do forno e do revestimento refratário, com o intuito de obter uma visão representativa. Foram escolhidas três zonas, uma em cada região do forno, para a coleta da evolução de temperaturas. Cada uma das três regiões apresentam características específicas e trabalham com temperaturas diferentes. A Figura 3 apresenta um desenho esquemático do forno, ilustrando as zonas onde os termopares foram inseridos. 


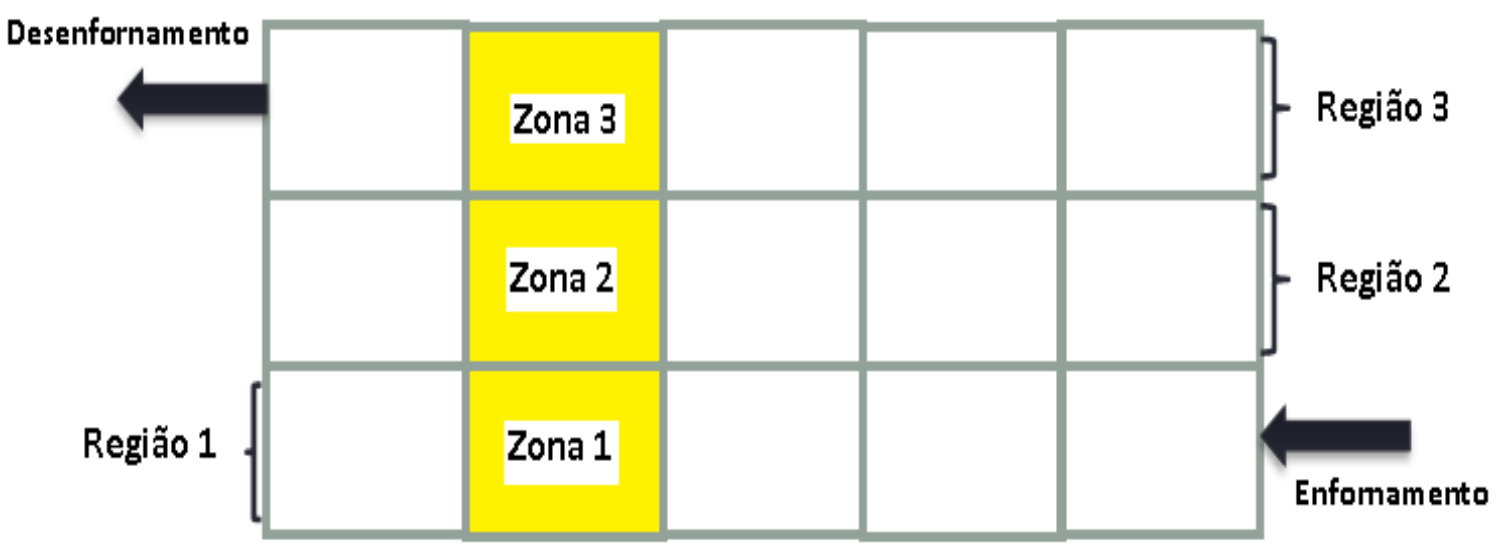

Figura 3. Desenho esquemático do forno com destaque para as zonas com termopares instalados.

Esta campanha de medição possibilitou uma boa visão geral da evolução de temperaturas. Os dados coletados foram utilizados para validação da simulação através de análise comparativa entre temperaturas reais e calculadas.

\subsection{Metodologia para a comparação entre os resultados da campanha de medição e resultados obtidos pelo simulador}

Para comparar os dados de temperatura coletados durante a campanha de medição com os resultados obtidos através da simulação, foram inseridas as configurações reais do forno durante o período de medição de temperaturas como dados de entrada do simulador. A partir destes dados, simulou-se a evolução das temperaturas dos pontos internos do revestimento refratário do teto e da soleira.

Os valores calculados e reais foram comparados através do cálculo de erro relativo. O valor de erro relativo foi avaliado levando em consideração sua representatividade de acordo com as características do processo de trabalho neste equipamento.

\subsection{Definição da condição de forno em regime quase permanente para reinício da produção}

O simulador foi utilizado para definição de um critério de forno preparado para receber tubos após um período improdutivo com redução de temperatura. Foram realizadas simulações, com dados de entrada reais, em situações de parada e retomada do regime de trabalho nas quais o equipamento havia deixado o estado de regime permanente. A partir da análise dos resultados foi definida a condição de forno em regime quase permanente, na qual a temperatura do revestimento apresenta pequena variação, que seria ideal para reinício da produção. Esta condição foi caracterizada pela temperatura interna mínima do refratário para início do processo produtivo com garantia de qualidade do processo de aquecimento dos tubos.

\subsection{Definição da estratégia de controle do forno visando redução de consumo}

A possibilidade de avaliar a evolução destas temperaturas para os diferentes cenários simulados permitiu a definição da condição de regime quase permanente do forno, onde as temperaturas do revestimento refratário são praticamente constantes ao longo do tempo. Adotaram-se então, estratégias de controle da 
evolução da temperatura da atmosfera do forno com menor consumo de energia, visando o controle das temperaturas internas do refratário para a garantia do forno em estado de regime quase permanente no instante de retomada da produção.

\section{RESULTADOS E DISCUSSÃO}

Os resultados relativos à validação do simulador, à utilização do mesmo para a definição do estado de regime quase permanente do forno para retomada do enfornamento de tubos, e para a definição de estratégias de controle de temperatura visando menor consumo; permitem avaliar a ferramenta de simulação como bem sucedida.

\subsection{Validação do simulador}

Considerando o teto do forno, para as zonas analisadas, os resultados apresentaram valores de temperatura calculados próximos aos valores medidos. Em média, o erro relativo máximo ao longo do período analisado foi menor que $10 \%$. Os resultados obtidos para a soleira apresentaram erro relativo um pouco maior que $30 \%$ para os pontos de maior profundidade (mais próximos à face quente). Este erro pode ser explicado por algumas simplificações assumidas no modelo matemático, como a utilização de uma capacidade térmica média para os materiais da soleira. Entretanto, é possível observar que a evolução das temperaturas reais e calculadas é semelhante, ou seja, seguem o mesmo perfil de evolução ao longo do tempo. Quando considerada a temperatura calculada pelo modelo para o ponto mais externo da soleira, mais próximo à face fria, os resultados apresentaram menor diferença entre modelo e temperatura real. $\mathrm{O}$ erro relativo foi, em média, menor que $10 \%$.

O perfil de evolução de temperaturas reais e calculadas para todos os pontos considerados foi semelhante. Os resultados confirmam que a camada mais externa da soleira é a que leva mais tempo para atingir estado quase invariável de temperatura devido à sua maior capacidade de isolamento térmica. Portanto, este foi considerado o fator crítico para a definição de regime quase permanente do forno. Como o erro apresentado pelos cálculos da temperatura mais externa da soleira foi menor que 10\%, considerando-se a complexidade do processo e a representatividade dos resultados, o erro relativo entre cálculos e realidade pode ser considerado pequeno. Desta forma, o simulador foi considerado validado.

\subsection{Condição de forno em regime quase permanente para reinício da produção}

Após certo tempo fora de regime de trabalho, observou-se através da campanha de medições temperatura, que o revestimento refratário apresentou um processo gradual de perda de temperatura. Com relação ao processo de elevação de temperatura do forno, visando sua preparação para entrar em regime de trabalho, foi observado comportamento gradual de elevação de temperatura até certo nível. A partir desse ponto, desde que não ocorresse mudança significativa nos níveis de temperatura de trabalho, toda a energia recebida pelo material refratário não refletia em variação de temperatura significativa, caracterizando-se assim o estado de regime permanente.

Com base nestas informações, a ferramenta de simulação foi utilizada para simular e analisar as temperaturas durante campanhas de produção que ocorreram após um 
período de forno fora de regime permanente. Durante período sem produção, o forno foi mantido em condição de baixa temperatura (forno desligado, ou conservado com temperatura menor que a temperatura normal de regime de trabalho). Observou-se que o processo produtivo no forno poderia ser retomado um pouco antes da entrada em regime permanente. A partir desta análise, definiu-se como critério para reinício da produção, um estado de regime quase permanente. Esta condição foi caracterizada através da definição de uma porcentagem mínima em relação à temperatura calculada para regime permanente da camada mais externa do refratário da soleira (a última a aquecer).

A definição da condição de regime quase permanente para reinício de produção possibilitou otimização do controle de temperatura do forno, proporcionando redução do consumo de energia, com garantia da qualidade dos produtos.

\subsection{Redução de consumo energético}

A utilização do simulador computacional para a definição da estratégia de controle de temperatura forno, com resfriamento e aquecimento controlados durante períodos improdutivos para a garantia do estado de regime quase permanente no instante de reinício da produção, permitiu a redução do consumo de energia. Considerando-se um período sem produção de dois dias, a redução de temperatura com base nas simulações proporcionou uma economia de aproximadamente $20 \%$ em relação ao volume de gás natural que seria necessário se o nível de temperatura de trabalho do forno fosse conservado.

\subsection{Ganhos com melhoria da qualidade da produção}

Os ganhos proporcionados pelo simulador desenvolvido com melhoria da qualidade dos produtos estão relacionados à utilização deste para retomada do processo produtivo com determinado material crítico após forno parado. Nesta situação, para garantir a qualidade dos produtos, eram utilizados setups de temperatura mais altos. A utilização da ferramenta de simulação proporcionou uma redução da temperatura de setup para estes materiais e permitiu uma redução anual no consumo de combustível de aproximadamente $\mathrm{R} \$ 50.000$.

\subsection{Apresentação da ferramenta}

A Figura 4 apresenta como a estrutura do simulador está organizada, com destaque para os dados de entrada e saída na ferramenta de simulação para um dos cenários de controle considerados no forno. Os dados apresentados não são reais e têm objetivo apenas demonstrativo. 


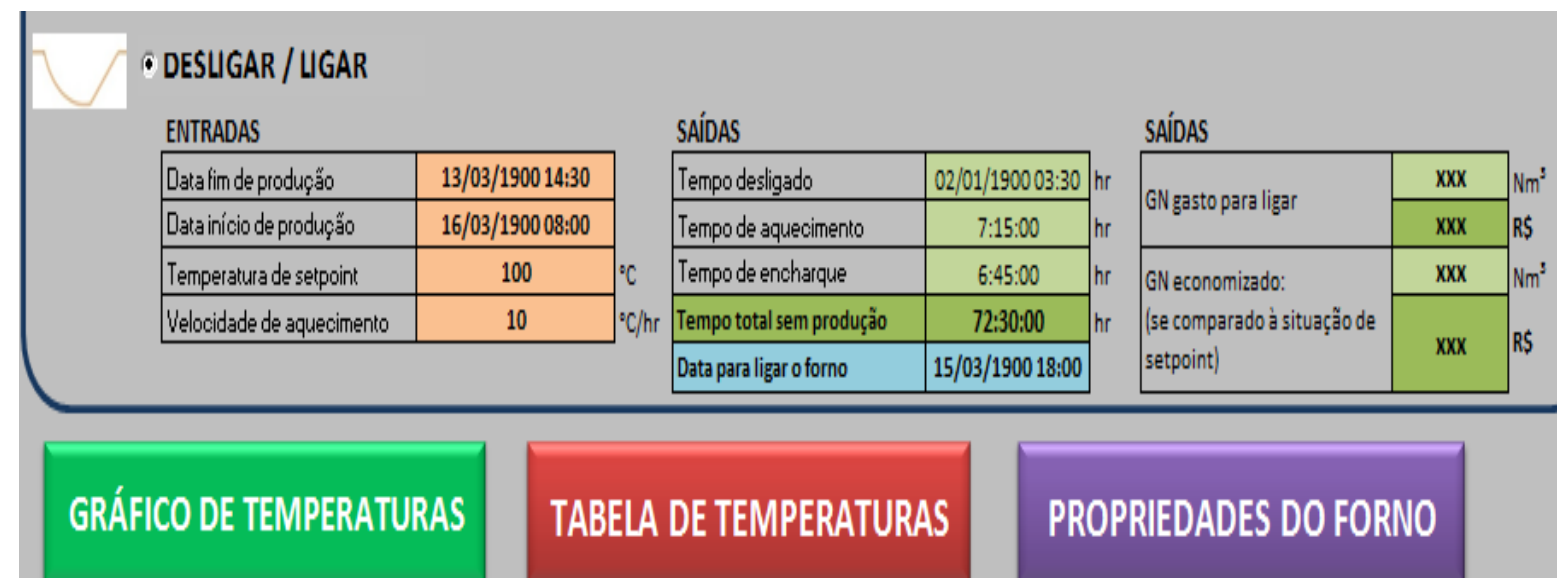

Figura 4 - Representação da ferramenta de simulação.

A Figura 5 ilustra os resultados gráficos de uma simulação realizada com dados irreais, apenas com o intuito de representar a aplicação da ferramenta desenvolvida. Pode ser observado na figura, o resfriamento e aquecimento da atmosfera do forno, os valores de temperatura calculados para os diferentes pontos internos do revestimento refratário e o valor da temperatura definida para caracterizar a condição de regime quase permanente do forno (destacada na figura).

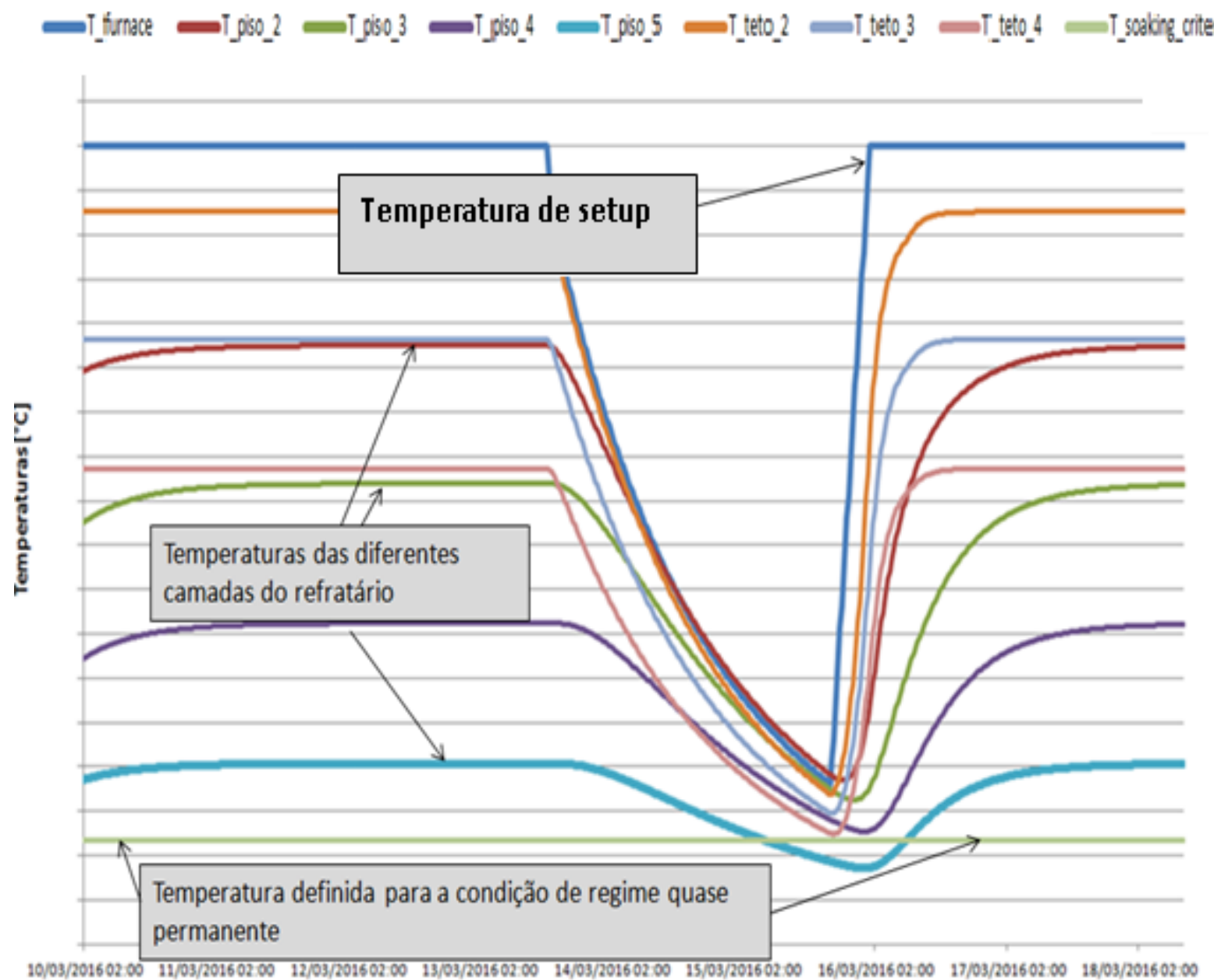

Figura 5 - Representação dos resultados gráficos 


\section{CONCLUSÃO}

O desenvolvimento desta ferramenta computacional de simulação permitiu analisar criticamente a evolução de temperaturas no forno durante períodos sem produção. Considerando os resultados de simulações, foi possível estudar melhores estratégias de controle de temperatura em diferentes situações. A utilização da ferramenta computacional para retomada do processo produtivo com determinado produto crítico após um período de forno parado, proporcionou economia de combustível de aproximadamente $\mathrm{R} \$ 50.000,00$. As novas estratégias de controle, com redução de temperatura durante períodos improdutivos, possibilitaram uma economia de aproximadamente $20 \%$ em relação ao volume de gás natural necessário para a manutenção do forno com mesmo nível de temperatura. 0 simulador apresentou importância estratégica para a redução do consumo de energia aliado à garantia da qualidade do aquecimento de tubos.

\section{Agradecimentos}

À empresa Vallourec Tubos do Brasil S.A. (VBR) por apoiar projetos de pesquisa e desenvolvimento.

À Dra. Lis Nunes Soares, engenheira especialista em processos térmicos, pela orientação do projeto.

À equipe de Processos Térmicos da Vallourec, Kássio Nogueira Cançado e Stephano Joviano Miranda, estudantes de engenharia mecânica; pelo auxílio em todas as fases do estudo.

A toda equipe dos setores de Tratamento Térmico, Combustão e Manutenção de Refratários da Laminação Contínua da VBR Usina Barreiro, pelo apoio e confiança no projeto.

\section{REFERÊNCIAS}

1 De Loze A.G., Rodrigues L. F. O. , Soares L.. "Desenvolvimento de modelo matemático para transferência de calor no revestimento refratário de um forno de tratamento térmico de tubos". Associação Brasileira de Metalurgia, Materiais e Mineração. (2016, No Prelo).

2 E.P. Keramidaa, H.H. Liakosa, M.A. Fountib, A.G. Boudouvisa,N.C. Markatosa. "Radiative heat transfer in natural gas-fired furnaces", International Journal of Heat and Mass Transfer 43 (2000).

3 Incropera, F.P... Dewitt, D.P. Fundamentos de Transferência de Calor e de Massa, 3a edição, LTC - Livros Técnicos e Científicos Editora S. A., R. J. 1990.

4 Mullinger Peter and Jenkins Berrie. Industrial and Process Furnaces. Principles, Design and Operation.

5 Taine J., lacona E., Petit J-P., Transferts Thermiques - Introduction aux transferts d'énergie, quatrième édition, Dunod. 